\section{Modes of coupled photonic crystal waveguides}

C. Martijn de Sterke

Centre for Ultrahigh-Bandwidth Devices for Optical Systems and School of Physics, University of Sydney, New South Wales 2006, Australia

\section{C. Botten and A. A. Asatryan}

Centre for Ultrahigh-Bandwidth Devices for Optical Systems and School of Mathematical Sciences, University of Technology Sydney, New South Wales 2007, Australia

\section{T. P. White and R. C. McPhedran}

Centre for Ultrahigh-Bandwidth Devices for Optical Systems, and School of Physics, University of Sydney, New South Wales 2006, Australia

Received December 23, 2003

We consider the modes of coupled photonic crystal waveguides. We find that the fundamental modes of these structures can be either even or odd, in contrast with the behavior in coupled conventional waveguides, in
which the fundamental mode is always even. We explain this finding using an asymptotic model that is valid

which the fundamental mode is always even. We explain th
for long wavelengths. 02004 Optical Society of America
OCIS codes: $130.2790,230.7370,050.1960$.

Coupled waveguides (CWs) occur in many optical devices. For example, the key element of directional couplers consists of two waveguides that are closely spaced to allow energy exchange. CWs have been studied both in conventional guided wave structures ${ }^{1}$ and in photonic crystals. ${ }^{2-4}$ The latter have received
much recent attention with the claim that short coupling lengths, the length over which energy couples
between the guides, can be achieved, providing the promise of compact devices.
protween, providing the An issue that has arisen is that of the bound modes of CWs. In symmetric conventional planar structures, the fundamental mode is even and the second mode is odd. ${ }^{1}$ The equivalent issue for photonic crystal waveguides, which does not affect their operation as a directional coupler, is not so well understood. Boscolo et $a .^{3}$ argued that the fundamental coupled waveguide mode (CWM) is even as in planar structures. However, here we show that for some structures the fundamental CWM is even or odd, depending on the guides' spacing. To illustrate the features of different geometries we use three examples, shown in Fig. 1. In all three cases we consider the polarization in which the electric field is orthogonal to the figure. The first [Fig. 1(a)] is conventional planar CWs. The second geometry riod $d$ ). Finally [Fig $1(c)$ we consider CWs in two dimensional photonic crystals with a square lattice of period $d$. The last two structures act only as waveguides and thus support only CWMs for frequencie within a bandgap of the periodic structure.

The analysis of the three structures initially proceeds in a common way. The key outcome of this analysis is Eq. (8) for all three structures; the reader mainly interested in results can proceed to this equation. We start the analysis by considering 0146.9592/04/121384-03\$15.00/0 the downward (-) propagating field at the top of the upper waveguide, indicated by $f_{1}$ (see Fig. 1). For the planar and layered structures, $f_{1}$ - gives the single phectic field component. For the two-dimensional photonic crystal $f_{1}{ }^{-}$is a vector of numbers $f_{1 p}{ }^{-}$corthe field's diffracted orders $p$, allowing the field to be written as

$E(x, y)=\sum_{p=-\infty}^{+\infty} \chi_{p}^{-1 / 2} f_{1 p}-\exp \left[-i \chi_{p}\left(y-y_{0}\right)+i \beta_{p} x\right]$.

Here $\beta_{p}=\beta_{0}+2 \pi p / d$ are the direction sines of these orders, $\chi_{p}=\left(k^{2}-\beta_{p}^{2}\right)^{1 / 2}$, where $k$ is the wave number, are the associated direction cosines, and $y_{0}$ is a reference plane, taken to be the top dashed line in

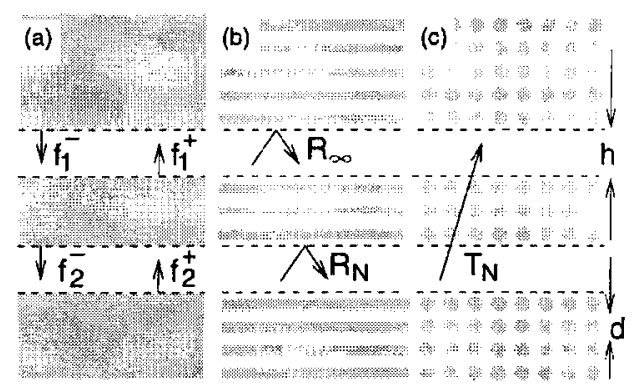

Fig. 1. Schematics of the geometries considered: (a) plaphotonic crystal with square lattice. For each geometry, the electric field is orthogonal to the plane, and the mode propagates in the plane.

- 2004 Optical Society
Fig. 1. Although in principle all orders, both propagating $\left(\left|\beta_{p}\right| \leq k\right)$ and evanescent $\left(\left|\beta_{p}\right|>k\right)$, need to be repres, $p$ the reflion off the semi-infinite struc tures surrounding the guides, is a scalar for the planar and the layered geometries and a square matrix for the two-dimensional photonic crystal. The same is true for $R_{N}$ and $T_{N}$, indicating the reflection and transmission, respectively, of the barrier between the guides, which consists of $N$ layers.

Given these definitions, we continue the analysis by relating the fields in Fig. 1 :

$$
f_{1}^{-}=R_{\infty} P f_{1}{ }^{+}, \quad f_{1}{ }^{+}=R_{N} P f_{1}^{-}+T_{N} P f_{2}{ }^{+},
$$$$
f_{2}{ }^{-}=T_{N} P f_{1}{ }^{-}+R_{N} P f_{2}{ }^{+}, \quad f_{2}{ }^{+}=R_{x_{0}} P F_{2}
$$

Here $P$ is the operator that propagates the field between the dashed lines in Fig. 1 over guide width $h$. Since the guides are uniform, the field can be written as a plane wave for the planar and the layered geometries and as a plane-wave superposition for the two-dimensional photonic crystal. In that case $P$ is a diagonal matrix with elements $\exp \left(i \chi_{p} h\right)$. By comshow that $f_{1}^{-}$ $U \mathrm{f}_{2}{ }^{+}$and $\digamma_{2}{ }^{+}=U f_{1}{ }^{-}$, where

$$
U=\left(I-R_{\infty} P R_{N} P\right)^{-1} R_{\infty} P T_{N} P,
$$

and $I$ is the unit operator. Therefore $\left(I-U^{2}\right) f_{1}{ }^{-}=$ 0 , and thus $(I-\sigma U) f_{1}^{-}=0$, where $\sigma= \pm 1$, and $f_{2}{ }^{+}=\sigma f_{1}{ }^{-}$. Thus, for this symmetric structure, the Eq. (3), we find

$I-\sigma U=\left(I-R_{\infty} P R_{N} P\right)^{-1}\left[I-R_{\times} P\left(R_{N}+\sigma T_{N}\right) P\right]$.

From the results of Botten et al., ${ }^{6}$ it is found that

$$
R_{N}=\left(R_{x}-Q^{N} R_{\infty} Q^{N}\right)\left(I-R_{\infty} Q^{N} R_{\infty} Q^{N}\right)^{-1},
$$$$
T_{N}=\left(I-R_{\times}{ }^{2}\right) Q^{N}\left(I-R_{\propto} Q^{N} R_{\alpha} Q^{N}\right)^{-1} \text {, }
$$

where $Q$ expresses how the Bloch functions of the periodic structure transfer through a layer. Since the
Bloch functions are the eigenfunctions of the structure, $Q$ depends on $\mu_{i}$, the eigenvalues of the translation operator. For propagating Bloch functions $\mu$ lies on the unit circle, whereas for evanescent modes $|\mu|<1{ }^{5}$ The correctness of Eqs. (5) for planar structures can be ascertained by comparison with the results for a Fabry-Perot interferometer that were presented in
Ref. 6. For a mode to exist, we require $I-\sigma U$ to be Ref. 6. For a mode to exist, we require $I-\sigma U$ to
singular, which by Eqs. (4) and (5) is equivalent to

$\operatorname{det}\left[I-R_{\infty} P\left(R_{\infty}+\sigma Q^{N}\right)\left(I+\sigma R_{\infty} Q^{N}\right)^{-1} P\right]=0$

\section{(6)}

From here on, the exact treatments of the three geometries differ, as the different natures of the fields become important. However, we use an approxima-
tion for the two-dimensional photonic crystal that lets us consider all three geometries on the same footing. This approximation is valid for long wavelengths, exists and all single propagating difraction order physics is then dominated by the single propagating order $(p=0)$ so all evanescent orders $(p \neq 0)$ can be dropped. Since this approximation, in which all

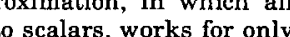
long wavelengths, it is valid for frequencies up to and including most of the first bandgap of the twodimensional photonic er ystal. In the long-wavelength approximation all quantities commute, and Eq. (6) reduces to

$$
R_{x}^{2} P^{2} \frac{1+\sigma \mu^{N} / R_{\infty}}{1+\sigma \mu^{N} R_{\infty}}=1
$$

where $\mu$, which is now real, represents the decay of the field over a lattice period. If $\mu<0$, the field changes ign after a period, similar to the behavior at the edge field over a period is unchan $\mu$, in the Brillouin ene center The planar structure in $\mathrm{Fig}$ (1) which has no periodicity, can be associated with $\mu>0$, since evanescent fields do not change sign. Now in a gap $\left|R_{\infty}\right|=1$, and since $|P|=1$, Eq. (7) can, for all three geometries, be written as

$$
\chi_{0} h+\arg \left(R_{\infty}+\sigma \mu^{N}\right)=m \pi .
$$

Here $m$ is an integer, and $\chi_{0}$ is the direction sine of the propagating order. The $\sigma \mu^{N}$ term characterizes the barrier between the guides, since, when the guides are widely spaced $(N \rightarrow \infty)$, the term disappears and the
equation for a single guide results.

$N_{N}$. key the sign of $\sigma \mu^{N}$ : when $\mu>0$, its sign is determined by that $\sigma$, and when $\mu<0$, the sign also depends on the
number of periods separating the guides, $N$. Consider first the planar geometry in Fig. 1(a), for which $\mu>0$ and, since it relies on total internal reflection, $-\pi<$ $\arg \left(R_{\infty}\right)<0 .{ }^{6}$ Then, compared with the parameters for a single guide, $\arg \left(R_{\infty}+\sigma \mu^{N}\right)$ increases for $\sigma>0$ (even mode), and thus for Eq. (8) to be satisfied $\chi_{0}$ must decrease and propagation constant $\boldsymbol{\beta}_{0}$ must increase. In contrast, when $\sigma<0, \beta_{0}$ decreases. Since the fundamental mode is that with the largest propagation constant at fixed frequency, the fundamental mode is even and the second mode is odd for a planar structure.

For the layered structure in Fig. 1(b) the sign of $\mu$ is not fixed. In the fundamental gap, and al aps $\mu>0$. Briefly, this is because the odd rape are narrowest at the edse of the Brillouin zone, where the fields change sign after a period, while the even gaps are narrowest at the Brillouin zone center, where the sign is unchanged. If, for the structure in Fig. 1(b), $-\pi<\arg \left(R_{x}\right)<0$, in the even gaps the behavior is as in the planar structure: the fundamental CWM is always even. But in the odd gaps the fundamenodd). We have found that at long wavelengths the 
1386 OPTICS LETTERS / Vol. 29, No. 12 / June 15, 2004

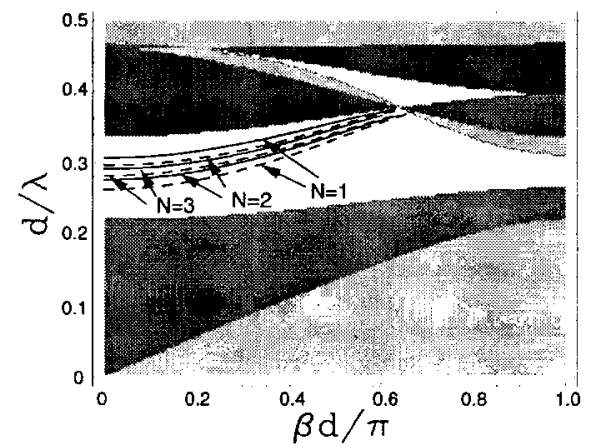

Fig. 2. Projected band structure for a two-dimensional bulk photonic crystal with parameters given in the text. bulk photonic crystal with parameters given in the text.
The dark-shaded regions indicate bands, the white regions The dark-shaded regions indicate bands, the white regions
indicate gaps with $\mu<0$, and the light-shaded regions indicate gaps with $\mu>0$. CWM dispersion relations are also given for (solid curves) even and (dashed curves) odd modes.
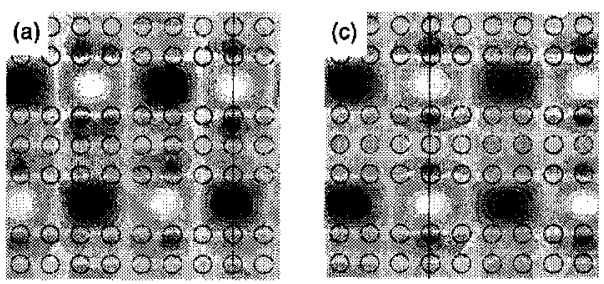

(b)

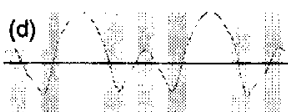

Fig. 3. Electric field in a two-dimensional photonic crystal with two coupled waveguides. (a), (b) odd CWM; (c), (d) even CWM. (a), (c) electric field contours; (b) and (d) field profiles through vertical lines in (a) and (c), respectively. The dark regions indicate the cylinders.

two-dimensional geometry in Fig. 1(c) behaves similarly to layered structures: in much of the lowest gap, where our approximation is valid, $\mu>0$, as illustrated in Fig. 2. This figure, calculated numerically without approximations, ${ }^{5}$ shows the projected band structure of a two-dimensional photonic crystal with a square lattice and cylindrical inclusions with radius $a=0.3 d$ and refractive index $n=3$ in a background of $n=1$. Also indicated are dispersion curves for the CWMs for two waveguides of width $h=d$, and $N=1,2,3$; for $N=1$ and $N=3$ the fundamental mode is odd, and for $N=2$ it is even, consistent with our discussion.

Figures 3 show the field of the structure in Fig. 2 and $N=3$ at wavelength $\lambda=3.05 d$. In the bright regions in Figs. 3(a) and 3(c), the electric field has a positive phase, and in the dark regions it is negative Thus Figs. 3 (a) and $3(\mathrm{~b})$ refer to the odd CWM, for which $\mu=-0.465$, Figs. 3(c) and 3(d) refer to the even CWM, for which $\mu=-0.487$. Note from Figs. 3(a) and $3(\mathrm{~b})$ that the latter has a shorter period, and the odd CWM therefore has the largest propagation constant and is thus the fundamental CWM. Indeed, the propagation constants of the odd and even CWMs are $\beta_{o} d=1.353$ and $\beta_{e} d=1.239$, respectively.

It is well known that, for structures in which the refractive index varies in one direction [Figs. 1(a) and 1(b)], the oscillation theorem applies, according to which the $n$th eigenfunction has $n-1$ nodes. ${ }^{7}$ This is consistent with the results for the planar structure [Fig. 1(a)], since an odd mode has at least one node and thus cannot be fundamental. The argument for the layered structure [Fig. 1(b)] is more subtle since we are interested only in CWMs, whereas the oscillation theorem applies to all modes, at frequencies in the gaps and in the bands. The fundamental mode in this structure is at the bottom of the lowest band. Thus, although the fundamental CWM can be either even or odd and has many nodes, it has one fewer node than the second CWM. For two-dimensional periodic structures, the oscillation theorem does not strictly apply, although for the long wavelengths considered here it behaves approximately as a layered structure. This is consistent with Figs. $3(\mathrm{c})$ and $3(\mathrm{~d})$, which show that the fundamental, odd CWM has one fewer node than the even CWM.

In conclusion, the fundamental CWM in the lowest gap of a photonic crystal can be either even or odd, depending on the phase change on reflection off the bulk photonic crystal and the separation of the guides. This does not affect the operation of directional couplers for which only the beat length of the CWMs $2 \pi /\left|\beta_{e}-\beta_{0}\right|$ enters. Although the results here refer to a specific polarization, we have found quantitatively similar results for the other polarization.

This work was produced with the assistance of the Australian Research Council under the ARC Centres of Excellence program. C. M. de Sterke's e-mail address is desterke@physics.usyd.edu.au.

\section{References}

1. K. Iizuka, Elements of Photonics, Vol. 2 (Wiley, New York, 2002), Chap. 9

2. M. Koshiba, J. Lightwave Technol. 19, 1970 (2001).

3. S. Boscolo, M. Midrio, and C. G. Someda, IEEE J. Quantum Electron. 38, 47 (2002).

4. A. Sharkawy, S. Shi, D. W. Prather, and R. A. Soref, Opt. Express 10, 1048 (2002), http://www.opticsexpress.org.

5. L. C. Botten, N. A. Nicorovici, R. C. McPhedran, C. Martijn de Sterke, and A. A. Asatryan, Phys. Rev. E 64, 046603 (2001).

6. M. Born and E. Wolf, Principles of Optics, 6th ed. (Pergamon, Oxford, 1980)

7. L. D. Landau and E. M. Lifshitz, Quantum Mechanics (Non-Relativistic Theory), 3rd ed. (Pergamon, Oxford, 1977), Sec. 21. 